\title{
HuCOP1 contributes to the regulation of DNA repair in keratinocytes
}

\author{
B. Fazekas ${ }^{1}$ (D) M. P. Carty $^{2} \cdot$ I. Németh ${ }^{1}$. \\ L. Kemény ${ }^{1} \cdot$ M. Széll ${ }^{4,5} \cdot$ É. Ádám ${ }^{3}$
}

Received: 6 October 2016/Accepted: 2 December 2016/Published online: 19 December 2016

(C) Springer Science+Business Media New York 2016

\begin{abstract}
We have previously demonstrated that the E3 ligase Human Constitutive Photomorphogenic Protein (huCOP1) is expressed in human keratinocytes and negatively regulates $\mathrm{p} 53$. The MutS homolog 2 (MSH2) protein plays a central role in DNA MMR mechanism and is implicated in the cellular response to anticancer agents, such as cisplatin. Our aim was to clarify whether huCOP1 plays a role in DNA MMR by affecting MSH2 protein level in human keratinocytes. To define the role of huCOP1 in DNA mismatch repair, we determined whether huCOP1 affects MSH2 abundance. MSH2 protein level was detected by immunocytochemical staining using a keratinocyte cell line in which the expression level of huCOP1 was stably decreased (siCOP1). To investigate whether huCOP1 silencing influences cisplatin-induced cell death,
\end{abstract}

Electronic supplementary material The online version of this article (doi:10.1007/s11010-016-2901-0) contains supplementary material, which is available to authorized users.

\section{B. Fazekas}

barbara.fazekas8@gmail.com

1 Department of Dermatology and Allergology, Faculty of Medicine, University of Szeged, Korányi fasor 6, Szeged 6720, Hungary

2 Centre for Chromosome Biology, and Biochemistry, School of Natural Sciences, National University of Ireland Galway, University Road, Galway, Ireland

3 Institute of Plant Biology, Biological Research Centre, Hungarian Academy of Sciences, Temesvári krt. 62, Szeged 6726, Hungary

4 MTA-SZTE Dermatological Research Group, University of Szeged, Szeged 6720, Hungary

5 Department of Medical Genetics, Faculty of Medicine, University of Szeged, Somogyi Béla st. 4, Szeged 6720, Hungary control and siCOP1 keratinocyte cells were treated with increasing concentrations of cisplatin and cell viability was recorded after 48 and $96 \mathrm{~h}$. Stable silencing of huCOP1 in human keratinocytes resulted in a reduced level of MSH2 protein. huCOP1 silencing also sensitized keratinocytes to the interstrand crosslinking inducer cisplatin. Our results indicate that decreased huCOP1 correlates with lower MSH2 levels. These protein level changes lead to increased sensitivity toward cisplatin treatment, implicating that huCOP1 plays a positive role in maintaining genome integrity in human keratinocytes.

Keywords huCOP1 - MSH2 - Keratinocyte - Cisplatin · Genome stability

\section{Introduction}

Constitutive Photomorphogenic Protein 1 (COP1) was first identified as a central negative regulator of light-regulated development in Arabidopsis thaliana [1]. The human orthologue was identified in 2003 [2]. COP1 is a wellconserved E3 ubiquitin ligase that regulates various cellular functions, such as proliferation and survival, through ubiquitin-mediated protein degradation in mammals, including humans $[3,4]$. Several putative targets of mouse and human COP1 (huCOP1) have been identified, including COP1 itself, p53, JUN and ETS variant family members. Transfection studies in cancer cell lines have suggested that huCOP1 targets p53 for ubiquitylation and proteasomal degradation [5].

We have previously demonstrated that huCOP1 is expressed in human keratinocytes, regulates p53, and potentially plays a pathogenic role in basal cell carcinoma and/or in squamous cell carcinoma [6,7]. These data 
collectively suggest that huCOP1 may have a role in DNA damage repair, and deciphering its role in these processes may bring us closer to understanding the DNA repair mechanism in keratinocytes.

DNA mismatch repair (MMR) is an ancient and conserved mechanism that significantly contributes to the accurate preservation of genetic material. MMR maintenance of genomic integrity is performed by correcting replicative mismatches (nucleotide mispairs, insertion/ deletion loops) that escape DNA polymerase proofreading [8]. The involvement of the mutS homolog 2 (MSH2) protein in DNA MMR is well characterized. MMR activity begins with mismatch recognition either by MutS $\alpha$, a heterodimer of MSH2 and MSH6 proteins, or by MutS $\beta$, a heterodimer of MSH2 and MSH3 [9].

MutS $\alpha$ proteins are degraded by the ubiquitin-proteasome pathway in a cell-type-dependent manner, indicating that one or several regulator(s) may interfere with huMutS $\alpha$ protein ubiquitination and degradation. Loss or depletion of MutS $\alpha$ from cells leads to microsatellite instability [10-13].

Several well-defined interactor molecules effecting MSH2 stability and/or activity are known: protein kinase C (PKC) is involved as a positive regulator of MMR activity, and the atypical PKC zeta regulates ubiquitination, degradation, and levels of huMutS $\alpha$ proteins. PKC zeta interacts with huMSH2 and huMSH6 proteins and phosphorylates both [14]. It has also been published that MSH2 interacts with several class I and II histone deacetylases (HDAC). HDAC6 deacetylates and ubiquitinates MSH2, leading to MSH2 degradation and reduced cellular sensitivity to DNA-damaging agents [15]. Namdar et al. [16] recently demonstrated that selective inhibition of HDAC6 induces DNA damage and sensitizes transformed cells to anticancer agents. In contrast, other publications report on hypersensitivity of MutS $\alpha$ protein-depleted cells to DNA interstrand crosslink-inducing (ICL) agents [11-13].

An increasing body of evidence suggests the role of huCOP1 in genome integrity, e.g., huCOP1-mediated p53 degradation was impaired in response to DNA damage, allowing p53 stabilization and activation [5, 17-19]. Based on our previous results with huCOP1 expression and function in keratinocytes, we hypothesized that this molecule is involved in the maintenance of genome integrity. Therefore, we initiated a set of experiments to investigate whether huCOP1 has a role in the regulation of MSH2 abundance in human keratinocytes.

In this paper, we describe that decreased huCOP1 level is correlated with downregulated $\mathrm{MSH} 2$ levels in human keratinocytes. Moreover, we provide data on increased sensitivity of keratinocyte cells to cisplatin treatment that results from decreased huCOP1 level.

\section{Materials and methods}

\section{Cell culture}

A HPV-immortalized human keratinocyte cell line (HPVKER clone II/15), in which TP53 is intact, was used for the establishment of the siCOP1 cell line [20]. The keratinocyte cell lines used in the experiments-control and siCOP1, in which the expression level of huCOP1 was stably decreased-have been described previously [7]. HPV-KER cells were maintained in keratinocyte serumfree medium (Gibco ${ }^{\circledR}$ Keratinocyte SFM Kit; Life Technologies, Copenhagen, Denmark) supplemented with 1\% antibiotic/antimycotic solution (PAA, Pasching, Austria) and $1 \%$ L-glutamine (PAA) at $37{ }^{\circ} \mathrm{C}$ in a humidified atmosphere containing $5 \% \mathrm{CO}_{2}$. The medium was changed every 2 days.

\section{Immunocytochemistry}

Control and siCOP1 keratinocytes were grown on culture slides (BD Falcon, Bedford, MA, USA) and immunostained $48 \mathrm{~h}$ after seeding. Immunocytochemistry was carried out using a previously described procedure [7]. As primary antibody, the mouse monoclonal anti-human MSH2 antibody was used at a dilution of 1:50 (product no. IR08561, Clone FE11, Dako, Denmark). After rinsing with TBS, cells were incubated with Alexa Fluor 647-labeled anti-mouse secondary antibody produced in goat (Invitrogen, Carlsbad, CA, USA) at a dilution of 1:400 for $3 \mathrm{~h}$ in the dark at room temperature. The subsequent semiquantitative analysis was carried out using the Metamorph software (Universal Imaging Corp., Sunnyvale, CA, USA).

\section{Real-time qRT-PCR experiments}

Total RNA was isolated from control and siCOP1 cells using the Direct-zol ${ }^{\mathrm{TM}}$ RNA MiniPrep (Zymo Research Corporation, Irvine, CA, USA) according to the manufacturer's instructions. cDNA was synthesized from $5 \mu \mathrm{g}$ total RNA with the Maxima First Strand cDNA Synthesis Kit for RT-PCR (Thermo Scientific, Waban, MA, USA).

Real-time qRT-PCR experiments were carried out with the Universal Probe Library system (F. Hoffmann-La Roche AG, Basel, Switzerland). Sequences of the primers used for PCR amplification:

MSH2:

FWD: CCAGCAGCAAAGAAGTGCTA; REV: GCA AAATGAGGCACTGGTCT; UPL probe No: 21;

18S: 
FWD: CGCTCCACCAACTAAGAACG; REV: CTCA ACACGGGAAACCTCAC; UPL probe No: 77.

\section{Immunoprecipitation}

Ubiquitinated proteins were immunoprecipitated from total cell lysates (1.0 million cells) using the Immunoprecipitation Kit Protein G (Roche Applied Science, Penzberg, Germany) and anti-human ubiquitin mouse primary antibody in $50 \mu \mathrm{l}$ final volume (cat. no. sc-52750, Santa Cruz Biotechnology Inc., Heidelberg, Germany). Parallelly, MSH2 antibody produced in mouse (Abcam, Cambridge, UK) or HDAC6 antibody produced in rabbit (Santa Cruz Biotechnology Inc., Heidelberg, Germany) were also used for immunoprecipitation. Immunoprecipitated proteins were size separated on a $10 \%$ SDS-polyacrylamide gel, and western blots were performed using MSH2 or HDAC6 antibody at 1:500 or 1:200 dilution to detect these proteins of the loaded samples. Alkaline phosphatase-conjugated anti-mouse or anti-rabbit IgG (Sigma-Aldrich, St. Louis, MO, USA) were used as secondary antibodies and the blots were developed using 5-bromo-4-chloro-3-indolyl phosphate/nitroblue tetrazolium as substrate.

\section{Cell viability assay}

Cell viability was assessed using the XTT assay (Roche, Basel, Switzerland). This method is based on the fact that metabolically active cells cleave the yellow tetrazolium salt XTT (2,3-bis-(2-methoxy-4-nitro-5-sulfophenyl)-2Htetrazolium-5-carboxanilide) which then forms an orange formazan dye. The amount of formazan dye directly correlates with the number of metabolically active cells [21, 22].

For treatment with cisplatin $(1 \mathrm{mg} / \mathrm{ml}$ solution, Ebewe Pharma, Vienna, Austria), cells were seeded in triplicate on a 96-well plate at $7.5 \times 10^{3}$ cells per well in $150 \mu \mathrm{l}$ media. After $24 \mathrm{~h}$ of incubation in a humidified incubator at $37{ }^{\circ} \mathrm{C}$ and $5 \% \mathrm{CO}_{2}$, media was removed and cells were treated with the indicated doses of cisplatin $(0.5-10 \mu \mathrm{M})$ in fresh medium [23]. After $24 \mathrm{~h}$ of incubation, media containing the cisplatin was removed, cells were washed twice with PBS, and the cells were allowed to recover in $150 \mu \mathrm{l}$ fresh media for 2 or 4 days. Immediately before use, a XTT labeling mastermix solution was prepared as recommended by the manufacturer (Roche, Dublin, Ireland), and $150 \mu \mathrm{l}$ mastermix was pipetted into each well of the 96-well tissue culture plate. Cells were incubated for a further $4 \mathrm{~h}$. The absorbance was then measured at $490 \mathrm{~nm}$ using a Victor ${ }^{2}$ 1420 Multilabel Counter (Wallac, MA, USA). Results were expressed as the percentage viability relative to the viability of untreated cells.

\section{Results}

\section{HuCOP1 is implicated in the regulation of MSH2 protein level in human keratinocytes}

The important role of MSH2 in DNA MMR processes is well known [9]. Our first goal was to reveal whether huCOP1 affects MSH2 protein abundance in human keratinocytes. For this purpose, we used a well-characterized cell line in which the expression level of huCOP1 was stably decreased (siCOP1) [7]. The MSH2 protein in siCOP1 and control cells was visualized by immunocytochemical staining. We detected a significant decrease $(80 \%)$ in the MSH2 protein level in the siCOP1 cells compared to the control cells (Fig. 1a, b). Having detected a difference in $\mathrm{MSH} 2$ protein expression by immunocytochemistry, the question occurred if this was a consequence of differential regulation at the RNA or protein level. To clarify this issue, we carried out quantitative RT-PCR analysis and measured the MSH2 mRNA levels in the control and siCOP1 cell lines (Fig. 1c). We detected approximately 1.8-fold higher level of MSH2 transcripts in the siCOP1 line indicating that the decreased MSH2 protein abundance is not due to transcriptional downregulation of the MSH2 gene in the siCOP1 cell line.

We also investigated huCOP1 regulation of $\mathrm{MSH} 2$ protein level in human keratinocytes. It is well established that the ubiquitin-proteasome pathway is involved in the regulation of $\mathrm{MSH} 2$ protein expression in the U937 (monocytic), HL-60 (myelocytic), HeLa (epithelial), and MRC-5 (fibroblast) human cell lines [24]. Since huCOP1 functions as an E3 ubiquitin ligase, we compared the ubiquitination of the MSH2 protein in the siCOP1 and control cells. We immunoprecipitated the ubiquitinated proteins from total protein extracts using an anti-ubiquitin antibody, and then detected the amount of MSH2 protein in the precipitate by western blot analysis. These experiments revealed that ubiquitinated MSH2 protein was not detectable in human keratinocytes. To confirm that the lack of detection of the ubiquitinated MSH2 was not a result of a technical failure, we included the HCD6 protein as a positive control in the immunoprecipitation experiments (Supplementary Fig. 1).

\section{Silencing of huCOP1 sensitizes keratinocytes to the interstrand crosslinking inducer cisplatin}

MSH2 protein is a member of the DNA MMR pathway contributing to the cellular response to DNA damage [9]. $\mathrm{MSH} 2$ is implicated in the cellular response to anticancer agents, such as cisplatin, a DNA interstrand crosslink-inducing (ICL) agent [10-13]. Based on the results of our 
Fig. 1 Determination of MSH2 mRNA and protein expression in siCOP1 and control cells. a $\mathrm{MSH} 2$ protein levels of control and siCOP1 cells were detected by

immunocytochemical staining (magnification, $\times 20$ ) and b subjected to semiquantitative analysis. c Relative MSH2 transcript levels in siCOP1 cells compared to the control cells measured by real-time RT-PCR analysis. Values reflect the gene expression changes in siCOP1 cells compared to the control cells. Expression levels were normalized to the $18 \mathrm{~S}$ ribosomal RNA. The average of three independent experiments is shown. Black bars control cells; gray bars siCOP1 cells
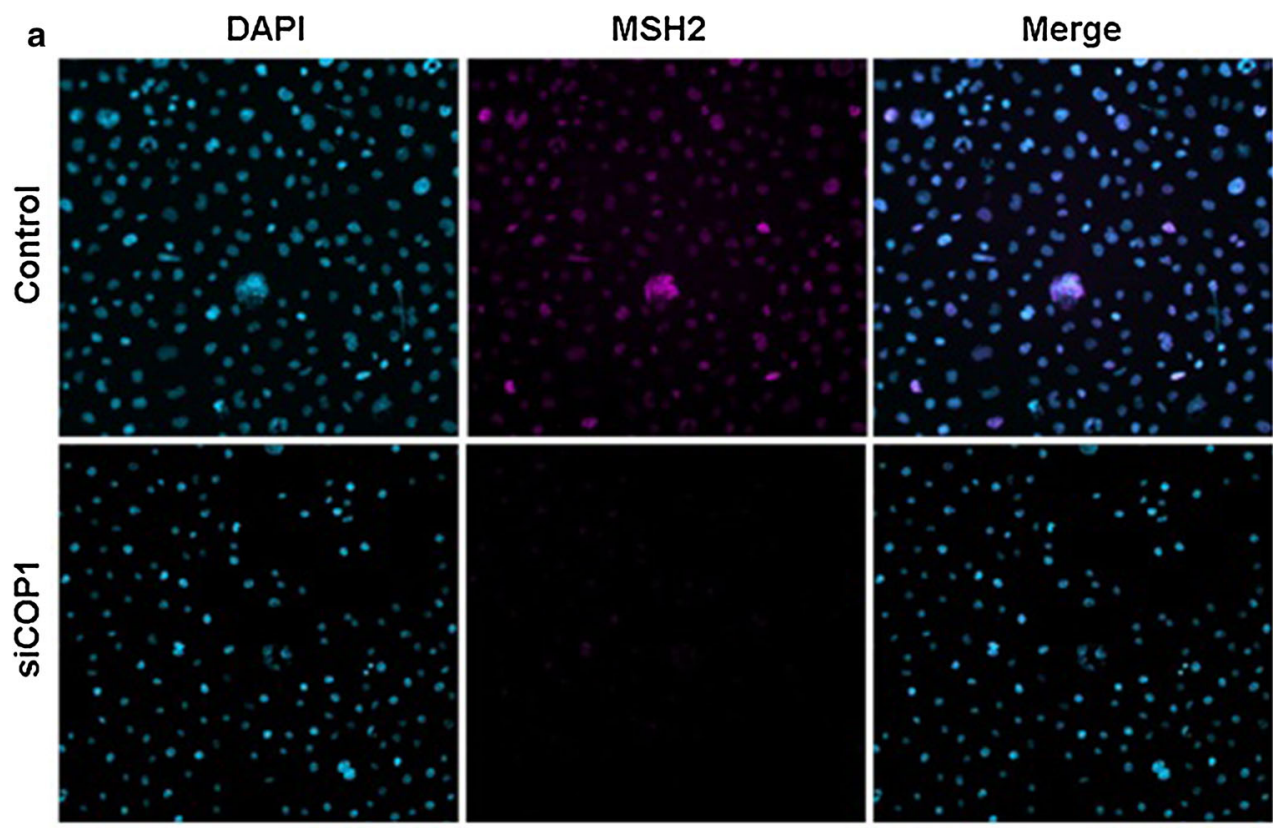

b

1,2

*

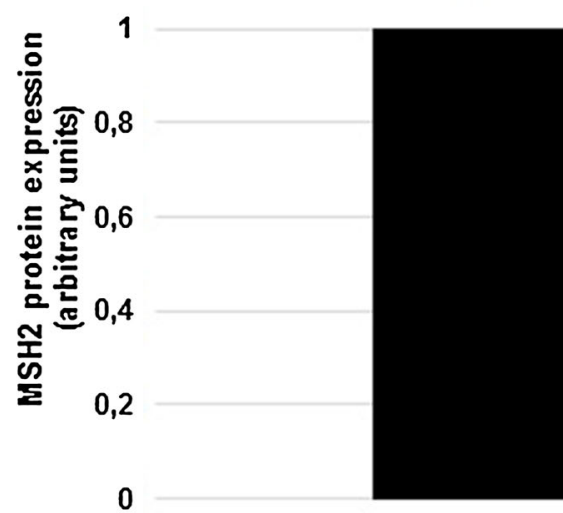

3

C

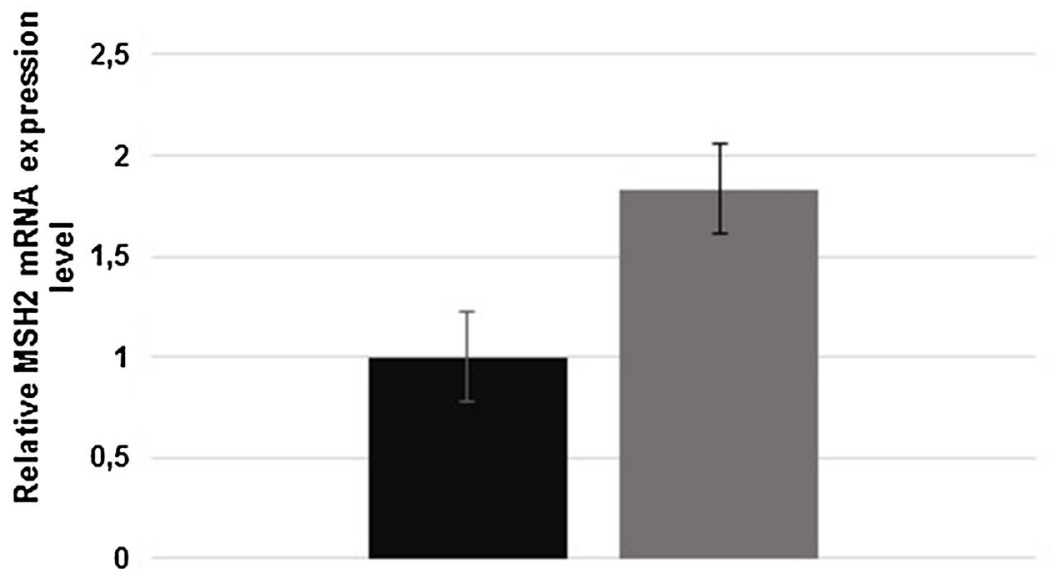




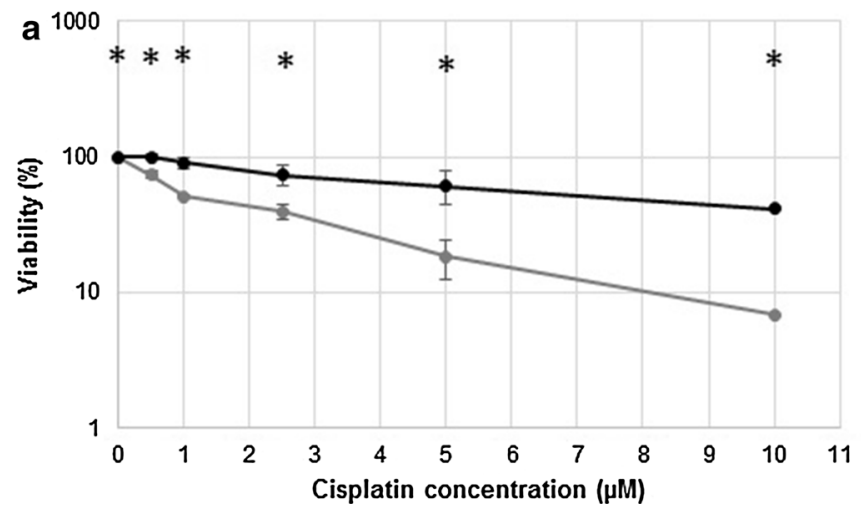

Fig. 2 Sensitivity of siCOP1 and control cells to cisplatin. a Average survival of siCOP1 and control cells after 2 days and $\mathbf{b}$ after 4 days of treatment with $0.5-10 \mu \mathrm{M}$ cisplatin. Cell survival was determined by an XTT assay performed in triplicate. The bars represent the standard

protein analysis, we hypothesized that huCOP1 plays a role in the maintenance of genome integrity. We investigated whether huCOP1 silencing has an effect on cisplatin-induced cell death. To this end, control and siCOP1 cells were treated with increasing concentrations of cisplatin $(0.5-10 \mu \mathrm{M})$ and cell viability was recorded after 48 (Fig. 2a) and $96 \mathrm{~h}$ (Fig. 2b). We found that siCOP1 cells displayed a significant hypersensitivity when exposed to cisplatin: the reduced level of huCOP1 caused a sixfold decrease in cell survival $48 \mathrm{~h}$ after treatment, and a 12 -fold decrease $96 \mathrm{~h}$ after treatment with $10 \mu \mathrm{M}$ cisplatin. These results suggest that huCOP1 influences the ICL repair mechanism in keratinocytes by indirectly modulating the MSH2 protein level in the cells.

\section{Discussion}

The ubiquitin-proteasome system (UPS) has emerged as a key regulatory mechanism in DNA repair pathways and in genome maintenance. Consequently, de-regulation of this system may lead to the development of various cancers [25]. The huCOP1 protein, an E3 ubiquitin ligase, promotes ubiquitin-dependent protein degradation [26-29]. An increasing body of evidence points to the role of huCOP1 in the maintenance of genome integrity $[17,19,30,31]$. It has been previously demonstrated that huCOP1 is overexpressed in cancer cells and represses p53-dependent tumor suppression via the UPS [5].

MSH2 is a protein involved in DNA MMR, which plays an important role in the maintenance of genomic integrity by correcting replicative mismatches (nucleotide mispairs, insertion/deletion loops) that escape DNA polymerase proofreading [9].

To investigate the role of huCOP1 in DNA repair of human keratinocytes, we studied its effect on the

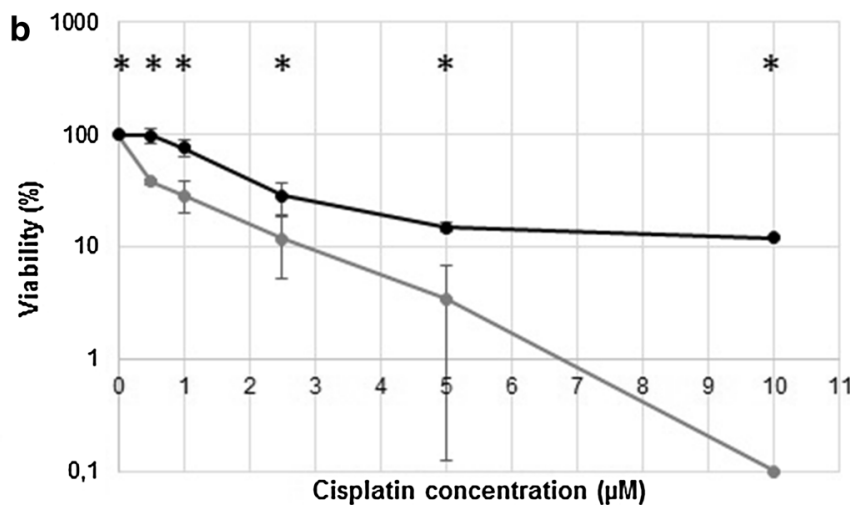

error of the means. Statistically significant differences between untreated and treated cells are indicated with asterisk $(p<0.05$, Student's two-tailed t test). Black line control cells; gray line siCOP1 cells

abundance of the MSH2 MMR protein in a well-characterized siCOP1 human keratinocyte cell line. Our data revealed that the level of $\mathrm{MSH} 2$ protein was decreased in the siCOP1 cells. The slightly elevated MSH2 mRNA level detected in these cells indicates also that huCOP1 is not implicated in the transcriptional regulation, but it effects the MSH2 protein abundance in human keratinocytes. The ubiquitin-proteasome complex is responsible for the degradation of MutS $\alpha$ proteins, and thus for the ubiquitination of MSH2 in Saccharomyces cerevisiae, U937, HL60, HeLa, and MRC-5 cell lines [24, 32]. Arlow and coworkers proposed that monomeric MSH2 is targeted by different ubiquitin ligases [32]. Since huCOP1 is a wellknown E3 ubiquitin ligase, the question arose whether huCOP1 is able to influence MSH2 protein levels via the ubiquitin-proteasome system. To investigate if the decreased MSH2 level observed in siCOP1 cells is the consequence of the increased ubiquitination rate of the protein, we performed an immunoprecipitation assay using siCOP1 and control cells. In our experiments, ubiquitinated MSH2 protein was not detected in human keratinocytes. Similarly, Hernandez-Pigeon et al. [24] have described that they were not able to detect ubiquitinated $\mathrm{MSH} 2$ in epithelial cells and fibroblasts.

The fact that we could not detect ubiquitinated MSH2 protein in control and siCOP1 human keratinocytes indicates that huCOP1 does not modulate MSH2 protein levels directly by ubiquitination. It is well known that MSH2 can form heterodimers with MSH3 and MSH6 and that maintaining a constant ratio of the monomers is advantageous for cells [24]. It has been proven by genetic and biochemical approaches that the stoichiometry of MMR proteins is important. The possibility that MSH3 and/or MSH6 are ubiquitinated and the heterodimers subsequently undergo proteasomal degradation might explain the decreased level in keratinocytes of MSH2 that is not 
ubiqutinated. The function of the unidentified E3 ubiquitin ligase(s) and/or protease(s) acting in this process is likely partly inhibited by huCOP1. Similarly, the target of huCOP1 in these processes might be one of the E3 ligases that ubiquitinates MSH2 interacting partners, such as HDAC6 or PKC zeta.

MSH2 is implicated in the cellular response to DNA damage [9]. Many anticancer agents, such as cisplatin, induce DNA damage, primarily at guanine residues. This damage generates monoadducts, intrastrand or interstrand crosslinks in DNA, leading to the inhibition of DNA replication and transcription and ultimately to cell death $[33,34]$. Previous reports have suggested that MMR-defective cells are hypersensitive to ICLs [12, 13]. Although other reports, especially those dealing with the role of HDAC6 activity on MSH2 level, contradict this scenario $[15,16]$, the detailed mechanisms behind those phenomena are not known. It has been shown that the MSH2 level correlates with the activity of repair mechanisms in the cells. As we detected decreased MSH2 levels in siCOP1 keratinocytes, we investigated whether reduced huCOP1 abundance influences cisplatin-induced cell death. We found that siCOP1 cells displayed hypersensitivity when exposed to cisplatin, supporting a potential role of huCOP1 in the ICL repair mechanism.

Taken together, our results show that decreased huCOP1 levels correlate with lower MSH2 levels in keratinocytes. These protein level changes lead to increased sensitivity toward cisplatin treatment, implying that huCOP1 plays a positive role in maintaining genome integrity.

\section{Compliance with ethical standards}

Conflict of interest The authors declare that they have no conflict of interest.

\section{References}

1. Deng XW, Caspar T, Quail PH (1991) cop1: a regulatory locus involved in light-controlled development and gene expression in Arabidopsis. Genes Dev 5:1172-1182

2. Bianchi E, Denti S, Catena R, Rossetti G, Polo S, Gasparian S, Putignano S, Rogge L, Pardi R (2003) Characterization of human constitutive photomorphogenesis protein 1, a RING finger ubiquitin ligase that interacts with Jun transcription factors and modulates their transcriptional activity. J Biol Chem 278:19682-19690. doi:10.1074/jbc.M212681200

3. Marine JC (2012) Spotlight on the role of COP1 in tumorigenesis. Nat Rev Cancer 12:455-464. doi:10.1038/nrc3271

4. Wei W, Kaelin WG Jr (2011) Good COP1 or bad COP1? In vivo veritas. J Clin Invest 121:1263-1265. doi:10.1172/JCI57080

5. Dornan D, Wertz I, Shimizu H, Arnott D, Frantz GD, Dowd P, O'Rourke K, Koeppen H, Dixit VM (2004) The ubiquitin ligase COP1 is a critical negative regulator of p53. Nature 429:86-92. doi:10.1038/nature02514

6. Kinyo A, Kiss-Laszlo Z, Hambalko S, Bebes A, Kiss M, Szell M, Bata-Csorgo Z, Nagy F, Kemeny L (2010) COP1 contributes to
UVB-induced signaling in human keratinocytes. J Invest Dermatol 130:541-545. doi:10.1038/jid.2009.286

7. Fazekas B, Polyanka H, Bebes A, Tax G, Szabo K, Farkas K, Kinyo A, Nagy F, Kemeny L, Szell M, Adam E (2014) UVBdependent changes in the expression of fast-responding early genes is modulated by huCOP1 in keratinocytes. J Photochem Photobiol B 140:215-222. doi:10.1016/j.jphotobiol.2014.08.002

8. Charames GS, Bapat B (2003) Genomic instability and cancer. Curr Mol Med 3:589-596

9. Kolodner RD, Marsischky GT (1999) Eukaryotic DNA mismatch repair. Curr Opin Genet Dev 9:89-96

10. Aebi S, Kurdi-Haidar B, Gordon R, Cenni B, Zheng H, Fink D, Christen RD, Boland CR, Koi M, Fishel R, Howell SB (1996) Loss of DNA mismatch repair in acquired resistance to cisplatin. Cancer Res 56:3087-3090

11. Wu Q, Vasquez KM (2008) Human MLH1 protein participates in genomic damage checkpoint signaling in response to DNA interstrand crosslinks, while MSH2 functions in DNA repair. PLoS Genet 4:e1000189. doi:10.1371/journal.pgen.1000189

12. Wu Q, Christensen LA, Legerski RJ, Vasquez KM (2005) Mismatch repair participates in error-free processing of DNA interstrand crosslinks in human cells. EMBO Rep 6:551-557. doi:10. 1038/sj.embor.7400418

13. Fiumicino S, Martinelli S, Colussi C, Aquilina G, Leonetti C, Crescenzi M, Bignami M (2000) Sensitivity to DNA cross-linking chemotherapeutic agents in mismatch repair-defective cells in vitro and in xenografts. Int J Cancer 85:590-596

14. Hernandez-Pigeon H, Quillet-Mary A, Louat T, Schambourg A, Humbert O, Selves J, Salles B, Laurent G, Lautier D (2005) hMutS alpha is protected from ubiquitin-proteasome-dependent degradation by atypical protein kinase $\mathrm{C}$ zeta phosphorylation. J Mol Biol 348:63-74. doi:10.1016/j.jmb.2005.02.001

15. Zhang M, Xiang S, Joo HY, Wang L, Williams KA, Liu W, Hu C, Tong D, Haakenson J, Wang C, Zhang S, Pavlovicz RE, Jones A, Schmidt KH, Tang J, Dong H, Shan B, Fang B, Radhakrishnan R, Glazer PM, Matthias P, Koomen J, Seto E, Bepler G, Nicosia SV, Chen J, Li C, Gu L, Li GM, Bai W, Wang H, Zhang X (2014) HDAC6 deacetylates and ubiquitinates MSH2 to maintain proper levels of MutSalpha. Mol Cell 55:31-46. doi:10.1016/j.molcel. 2014.04.028

16. Namdar M, Perez G, Ngo L, Marks PA (2010) Selective inhibition of histone deacetylase 6 (HDAC6) induces DNA damage and sensitizes transformed cells to anticancer agents. Proc Natl Acad Sci USA 107:20003-20008. doi:10.1073/pnas.1013754107

17. Dornan D, Shimizu H, Mah A, Dudhela T, Eby M, O'Rourke K, Seshagiri S, Dixit VM (2006) ATM engages autodegradation of the E3 ubiquitin ligase COP1 after DNA damage. Science 313:1122-1126. doi:10.1126/science.1127335

18. Li DQ, Ohshiro K, Reddy SD, Pakala SB, Lee MH, Zhang Y, Rayala SK, Kumar R (2009) E3 ubiquitin ligase COP1 regulates the stability and functions of MTA1. Proc Natl Acad Sci USA 106:17493-17498. doi:10.1073/pnas.0908027106

19. Choi HH, Su CH, Fang L, Zhang J, Yeung SC, Lee MH (2015) CSN6 deregulation impairs genome integrity in a COP1-dependent pathway. Oncotarget 6:11779-11793. doi:10.18632/onco target. 3151

20. Polyanka H, Szabo K, Tax G, Goblos A, Agnes K, Tubak V, Ujfaludi Z, Boros I, Bata-Csorgo Z, Kemeny L, Szell M (2013) Characterization of UV-B induced cellular processes in a keratinocyte cell line (HPV-KER) immortalized with the HPV-E6 oncogene. J Investig Dermatol 133:S218-S218

21. Gerlier D, Thomasset N (1986) Use of MTT colorimetric assay to measure cell activation. J Immunol Methods 94:57-63

22. Scudiero DA, Shoemaker RH, Paull KD, Monks A, Tierney S, Nofziger TH, Currens MJ, Seniff D, Boyd MR (1988) Evaluation of a soluble tetrazolium/formazan assay for cell growth and drug 
sensitivity in culture using human and other tumor cell lines. Cancer Res 48:4827-4833

23. Cruet-Hennequart S, Villalan S, Kaczmarczyk A, O’Meara E, Sokol AM, Carty MP (2009) Characterization of the effects of cisplatin and carboplatin on cell cycle progression and DNA damage response activation in DNA polymerase eta-deficient human cells. Cell Cycle 8:3039-3050

24. Hernandez-Pigeon H, Laurent G, Humbert O, Salles B, Lautier D (2004) Degadration of mismatch repair hMutSalpha heterodimer by the ubiquitin-proteasome pathway. FEBS Lett 562:40-44. doi:10.1016/S0014-5793(04)00181-4

25. Schwartz AL, Ciechanover A (1999) The ubiquitin-proteasome pathway and pathogenesis of human diseases. Annu Rev Med 50:57-74. doi:10.1146/annurev.med.50.1.57

26. Wang H, Kang D, Deng XW, Wei N (1999) Evidence for functional conservation of a mammalian homologue of the light-responsive plant protein COP1. Curr Biol 9:711-714

27. Torii KU, McNellis TW, Deng XW (1998) Functional dissection of Arabidopsis COP1 reveals specific roles of its three structural modules in light control of seedling development. EMBO J 17:5577-5587. doi:10.1093/emboj/17.19.5577

28. Yi C, Wang H, Wei N, Deng XW (2002) An initial biochemical and cell biological characterization of the mammalian homologue of a central plant developmental switch, COP1. BMC Cell Biol $3: 30$
29. Yi C, Deng XW (2005) COP1—from plant photomorphogenesis to mammalian tumorigenesis. Trends Cell Biol 15:618-625. doi:10.1016/j.tcb.2005.09.007

30. Dornan D, Bheddah S, Newton K, Ince W, Frantz GD, Dowd P, Koeppen H, Dixit VM, French DM (2004) COP1, the negative regulator of $\mathrm{p} 53$, is overexpressed in breast and ovarian adenocarcinomas. Cancer Res 64:7226-7230. doi:10.1158/0008-5472. CAN-04-2601

31. Choi HH, Gully C, Su CH, Velazquez-Torres G, Chou PC, Tseng C, Zhao R, Phan L, Shaiken T, Chen J, Yeung SC, Lee MH (2011) COP9 signalosome subunit 6 stabilizes COP1, which functions as an E3 ubiquitin ligase for 14-3-3sigma. Oncogene 30:4791-4801. doi:10.1038/onc.2011.192

32. Arlow T, Scott K, Wagenseller A, Gammie A (2013) Proteasome inhibition rescues clinically significant unstable variants of the mismatch repair protein Msh2. Proc Natl Acad Sci USA 110:246-251. doi:10.1073/pnas.1215510110

33. Dronkert ML, Kanaar R (2001) Repair of DNA interstrand crosslinks. Mutat Res 486:217-247

34. McHugh PJ, Spanswick VJ, Hartley JA (2001) Repair of DNA interstrand crosslinks: molecular mechanisms and clinical relevance. Lancet Oncol 2:483-490. doi:10.1016/S14702045(01)00454-5 